\title{
Design of a 2 Mbps FSK near-field transmitter for wireless capsule endoscopy
}

\author{
J. Thoné*, S. Radiom, D. Turgis, R. Carta, G. Gielen, R. Puers \\ KU Leuven, ESAT-MICAS Department, Kasteelpark Arenberg 10, B-3001 Leuven, Belgium
}

\section{A R T I C L E I N F O}

\section{Article history:}

Received 26 September 2008

Received in revised form 16 October 2008

Accepted 30 November 2008

Available online $\mathrm{xxx}$

\section{Keywords:}

Capsule endoscopy

High data rate telemetry

FSK modulation

Near-field antenna

\begin{abstract}
A B S T R A C T
This paper introduces a novel and simple topology for an efficient, miniaturized, low power but high data rate transmitter, suitable for implanted devices. The application envisaged is in capsular endoscopy, where high quality images of the gastro-intestinal tract need to be transmitted continuously through the human body to an external receiver. A 2 Mbps Frequency Shift Keying transmitter is developed, consuming $2 \mathrm{~mW}$ at $1.8 \mathrm{~V}$, working at biocompatible frequencies.
\end{abstract}

(c) 2008 Elsevier B.V. All rights reserved.

\section{Introduction}

At present several wireless capsule endoscopy systems are available on the market (Given Imaging, Olympus EndoCapsule) [1]. Although appealing to the patient for comfort reasons, they lack three major properties: adequate image resolution $(256 \times 256$ pixels), sufficient frame rate $(2-7$ frames per second (fps)), and the ability to move around in a controlled way through the GI tract. These shortcomings hamper their breakthrough with gastro-enterologists, who still prefer the traditional endoscopes [2].

These limitations are a direct consequence of the finite energy supply available in these capsular endoscopes. All of them being battery powered, their lifetime is limited between 6 and $8 \mathrm{~h}$, consuming $25 \mathrm{~mW}$. The tight energy limit does not exist in the case of inductive powering [3,25].

Without the energy constraint, a higher resolution sensor and higher frame rates become possible. However, in order to get the mass of image data outside the body to the receiver, a high speed data transmitter is required.

\section{Requirements}

\subsection{Data rate}

Modern wired endoscopes are already equipped with High-Definition (HD) CCD cameras, providing up to $30 \mathrm{fps}$ at $1920 \times 1080$ pixels per frame [4]. This resolution would require

\footnotetext{
* Corresponding author. Tel.: +32 16321108; fax: +32 16321975.

E-mail address: jef.thone@esat.kuleuven.be (J. Thoné).
}

a raw Bayer data rate of $78 \mathrm{MByte} / \mathrm{s}$. CCD image sensors are not suitable for use in capsular endoscopes because of their high power consumption compared to their CMOS equivalent.

HD resolution in wireless endoscopy, even highly compressed, sounds like a fairy tale, for the simple reason that high data rate and low power is hard to combine. It is a questionable prognosis if the big advantage of patient comfort will surpass the need for HD image resolution.

Compared to the presently used $256 \times 256$ pixel resolution, a big improvement in image quality can already be obtained by using a $640 \times 480$ pixel (VGA) image sensor.

For $10 \mathrm{fps}$, which is a major step ahead of the current $2 \mathrm{fps}$, a raw Bayer data rate of 3.84 MByte/s is required.

Still being too high for low power transmission, appropriate compression algorithms have to be used to reduce the raw data rates to acceptable levels suitable for low power transmission.

\subsection{Compression}

Image compression basically removes visually redundant information from a picture or video stream, without exaggerated loss of detail or introduction of compression artifacts. The (lossy) compression algorithms are either based on removal of high frequency image content (e.g. JPEG) or on removing redundancy from the image colors [5]. A 20-fold compression can easily be reached without disturbing artifacts or visual image degradation. This will relax the data rate requirement to a more feasible $1.5 \mathrm{Mbps}$.

The choice of the compression engine is important at system level design: depending on the type of compression more bulky and power consuming RAM is needed. 


\subsection{Carrier frequency and modulation}

Little research has been done on the choice of the carrier frequency for through-body wireless near-field transmission.

The paper of Johnson and Guy describes the attenuation of electromagnetic (EM) waves through the human body [6]. This paper suggests to choose a relatively low $(<200 \mathrm{MHz})$ carrier, as the attenuation increases exponentially with the carrier frequency. The reduced attenuation would lead to less required transmitted power. It is currently not known if the same behavior can be expected for near fields, so it is hard to draw a definitive conclusion on the choice of frequency.

The work of Chirwa et al. suggests using a carrier between 450 and $900 \mathrm{MHz}$ for maximum radiation [7]. This work is based on finite-difference time-domain (FDTD) simulations on a human body model, and does not include antenna loading effects. The same antenna model was used for all frequencies, which partly accounts for the lower radiation intensities at lower frequencies. This work confirms the rapidly increasing absorption above $500 \mathrm{MHz}$, both for near- and far-fields, as described by Johnson [6].

The experiments of Wang et al. describe the in-vitro characterization of ingestible capsules for $30 \mathrm{MHz}$ and $868 \mathrm{MHz}$ [8]. They conclude that the low frequency capsule is less influenced by surrounding tissues, shows less orientation-dependent fading and a higher signal to noise $(\mathrm{S} / \mathrm{N})$ ratio for a certain power consumption.

The research suggests to use a carrier below $500 \mathrm{MHz}$, although there must exist a trade off between antenna size and wavelength. For far fields, a higher carrier frequency would require a smaller antenna, so less occupied space inside the capsule. As we are working in, or close to the near-field region (the transmission and reception antenna distance is smaller or equal to the wavelength), it is not sure whether this trade-off is still valid. Near-field simulations and experiments will lead to an optimal choice of the carrier.

Governmental (FCC and ERO) regulations will eventually define which frequency band can be used [9,10]. Candidates could be ISM (433.05-434.79 MHz) or MICS (402-405 MHz), although the defined bandwidth limits the maximum data rate.

Currently the $2 \mathrm{~m}$ amateur band ( $144 \mathrm{MHz}$ ) is used for data transmission, as this limits the interference with other important bands.

FSK is chosen as modulation type, for its simplicity in modulation and demodulation, and its inherent insensitivity to system non-linearities.

\subsection{Antennas}

The space around the transmitter antenna can be divided into two main regions as illustrated in Fig. 1: far field and near field.

In the far field, electric and magnetic fields propagate outward as an electromagnetic wave and are perpendicular to each other and to the direction of propagation. The angular field distribution does not depend on $r$, the distance from the antenna. The fields are

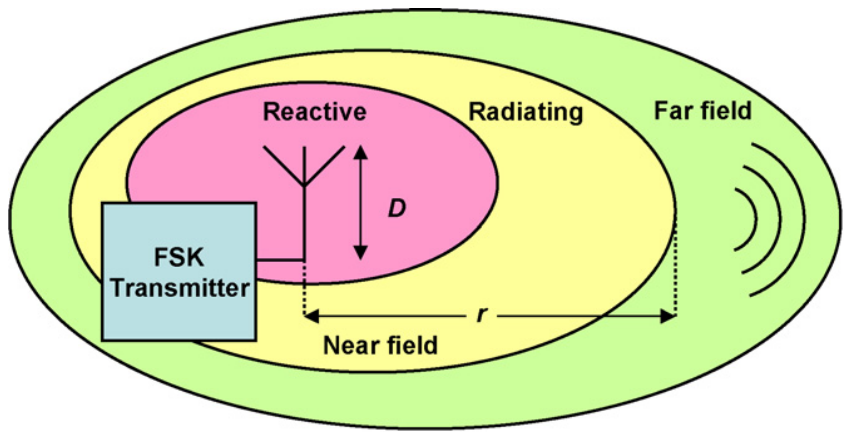

Fig. 1. Field regions transmitter antenna. uniquely related to each other via free-space impedance and decay as $1 / r$.

In the near field, the field components have different angular and radial dependence (e.g. $1 / r^{3}$ ). The near-field region includes two sub-regions: radiating, where the angular field distribution is dependent on the distance, and reactive, where the energy is stored but not radiated.

For antennas whose size is comparable to wavelength (as used in UHF RFID), the approximate boundary between the far-field and the near-field region is commonly given as $r=2^{*} D^{2} / \lambda$, where $D$ is the maximum antenna dimension and $\lambda$ is the wavelength. For electrically small antennas (as used in LF/HF RFID and in this application), the radiating near-field region is small and the boundary between the far-field and the near-field regions is commonly given as $r=\lambda / 2 \pi$.

When the receiver antenna is located in the near field of the transmitter antenna, the coupling between the antennas affects the impedance of both antennas as well as the field distribution around them. The equivalent antenna performance parameters (gain and impedance) can no longer be specified independently from each other and become position and orientation-dependent [11]. In general, to calculate the power received by the receiver in such a situation, one needs to perform a three-dimensional electromagnetic simulation of the near-field problem except when the transmitter is small and does not perturb the field of the receiving antenna.

The near field of a transmitter antenna can have several tangential and radial electric and magnetic field components which can all contribute to coupling. Two ultimate cases are magnetic (inductive) coupling and electric (capacitive) coupling.

In magnetic RFID systems, both receiver and transmitter antennas are coils, inductively coupled to each other like in a transformer. If the transmitter antenna is small, the coupling coefficient is proportional to [12]:

$C \propto f^{2} N^{2} S^{2} B^{2} \alpha$

where $f$ is the frequency, $N$ is the number of turns in transmitter coil, $S$ is the cross-section area of the coil, $B$ is magnetic field at the transmitter and $\alpha$ is the coil misalignment loss.

The primary coupling mechanism in near-field transmission can be either magnetic (inductive) or electric (capacitive). Depending on the environment, the field distribution can be affected by the presence of various objects. Inductive coupled systems, where most reactive energy is stored in the magnetic field, are mostly affected by objects with high magnetic permeability. The magnetic permeability of biological tissue is practically equal to the magnetic permeability of air.

On the other hand, capacitive coupling systems, where most reactive energy is stored in electric field, are affected by objects with high dielectric permittivity and loss. Since the body has high epsilon, it seems that inductive coupling is much more efficient for this kind of applications.

\section{Design strategy}

\subsection{Transmitter}

The first work on endoscopic telemetric capsules dates from 1957, where Mackay used a single transistor Hartley oscillator as transmitter [13]. The capsule was used to measure gastric pressures, where a pressure sensitive membrane connected to an iron core modulated the oscillator inductance and thereby the oscillation frequency. The operating frequency of this device was in the $100 \mathrm{kHz}$ range. 


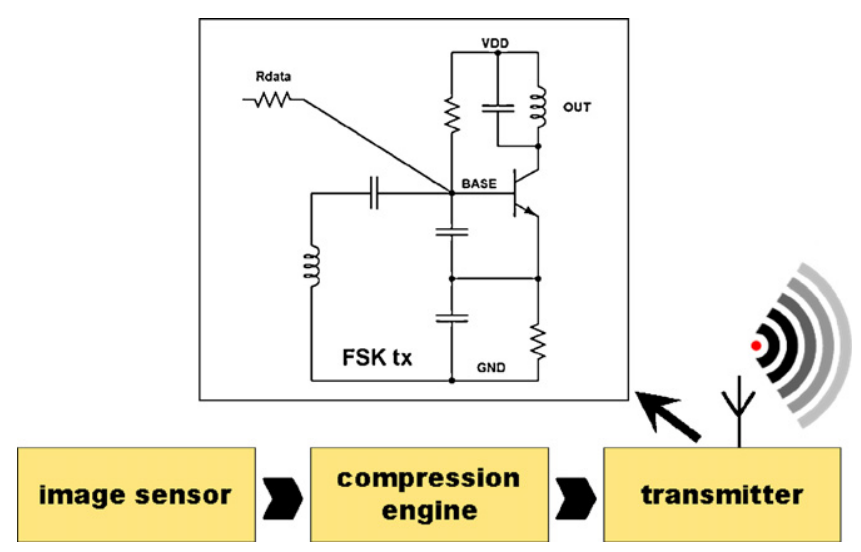

Fig. 2. Endoscopic capsule block diagram with $2 \mathrm{~mW} 2 \mathrm{Mbps}$ transmitter schematic.

The transmitter developed in earlier work [14] was redesigned such that the power consumption dropped with $66 \%$ while doubling the data rate. The transmitter consists of a single transistor Colpitts oscillator as shown in Fig. 2.The circuit is built up as a common collector circuit, with the antenna connected to the collector side. The presented topology operates the transistor with a unique double function: (1) it provides gain to the feedback loop to sustain the oscillation and (2) it provides a cascode function at the collector. During the capsule GI tract transit, the parasitic loads seen by the antenna change continuously. The cascode limits the antenna load detuning of the LC tank, which greatly improves frequency stability, and therefore reception. FSK modulation is achieved through modulation of the transistor base current. A change in the base current causes a change in base-emitter voltage, which influences the depletion layer width of the base-emitter junction. In this way the base-emitter capacitance is modulated, controlling the tuning of the LC tank.

The maximal data rate of this transmitter is limited by the RC time constant of the $R_{\text {data }}$ resistor and the capacitance seen at the base. It is clear that from a frequency higher than $1 /\left(R_{\mathrm{data}}{ }^{*} C_{\text {base }}\right)$, the modulation index decreases, because the injected base current is shorted in the base capacitance. Although the occupied bandwidth decreases, the $\mathrm{S} / \mathrm{N}$ ratio decreases too, and robust demodulation becomes more difficult at faster modulation rates.

From experiments, the limit was found to be at 2 Mbps.

As stated earlier in the requirements, it is more beneficial to use inductive near-field coupling for the transmitter-receiver system. For that purpose a coil transmitter antenna was developed, which was tuned to resonance around the transmitter frequency, using a trimmable capacitor. The tuned regime (for maximum power output) was measured using a spectrum analyzer. The antenna was designed as a proof of concept, but not simulated or optimized. For that reason further optimization regarding coupling, coil layout and matching is required in the future, when dedicated antennas will be designed and simulated.

\subsection{Receiver}

The receiver of [14] was reused, which is based on a SA639 IC application note. It consists of an IF mixing system used in DECT receivers. The FSK modulated carrier is converted back to a serial data stream by mixing the IF carrier with a phase-shifted version of itself. This results in a DC component containing the data and a component at twice the IF frequency, which is attenuated using a low pass filter.

A $2 \mathrm{~m}$ low noise amplifier (LNA) was built and included in the system, to improve the noise figure (NF) of the receiving system. The SA639 receiver has a NF of $11 \mathrm{~dB}$, where the LNA has NF of $3 \mathrm{~dB}$

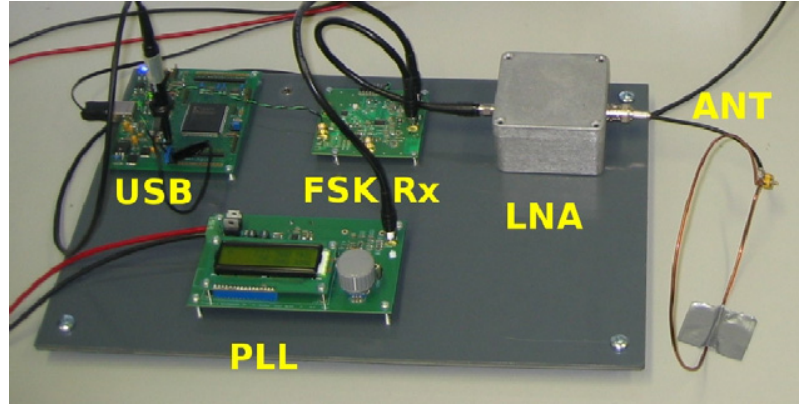

Fig. 3. FSK receiver setup showing USB interface, IF receiver, PLL, LNA and receiver antenna.

with a gain of $20 \mathrm{~dB}$. By including the LNA, the overall NF decreased with $6 \mathrm{~dB}$.

A tuned loop was used as receiving antenna, designed as a proof of concept for near-field reception.

The received data is resynchronized in a hardware developed data recovery system, written in VHDL and implemented in a Xilinx Spartan XC3S200. When a synchronization pattern for a new image frame is detected in the data stream, the data is buffered in a FIFO and sent through USB to a PC. On the PC the image data is visualized on the screen.

The receiver setup is depicted in Fig. 3, showing from left to right the USB interface + data recovery, the FSK demodulation board with the PLL below, and the LNA+ loop receiver antenna.

\section{Simulations}

The transmitter circuit was simulated using ELDO [23], in order to:

- optimize the component values to maximize the output power,

- optimize the component values for the correct carrier frequency,

- check for stable and fast start-up behavior,

- check the influence of parasitic (capacitive) load changes on the output,

- optimize the frequency deviation depending on the DATA input.

The sweep simulation depicted in Fig. 4 shows the output frequency at DC modulation. The typical frequency deviation between DATA $=0$ and DATA $=1$ is approximately $180 \mathrm{kHz}$.

The simulation results in Fig. 5 shows the modulated frequency of the transmitter with the DATA input toggling at $2 \mathrm{Mbps}$. The short nanosecond spikes are a result of the frequency measure-

\section{Carrier frequency vs VDATA}

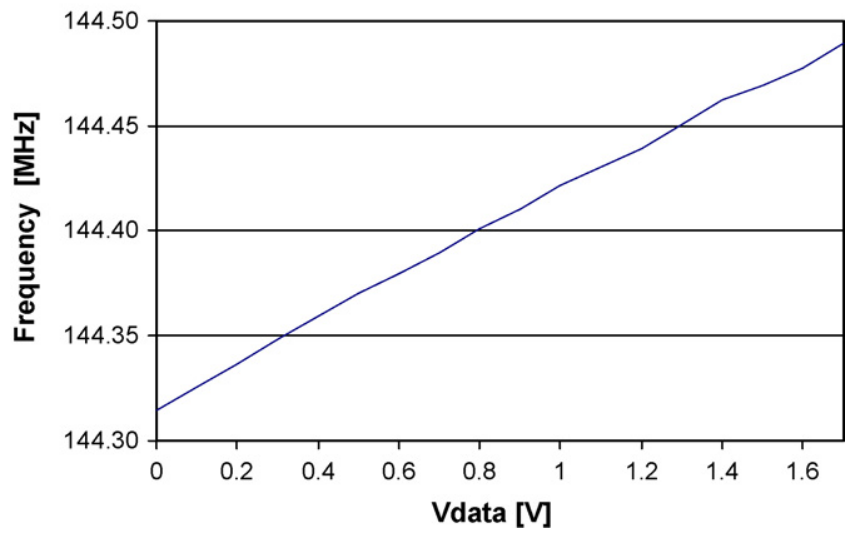

Fig. 4. Modulator frequency vs. voltage at DATA input simulation. 


\section{Output frequency deviation at $2 \mathrm{Mbps}$}

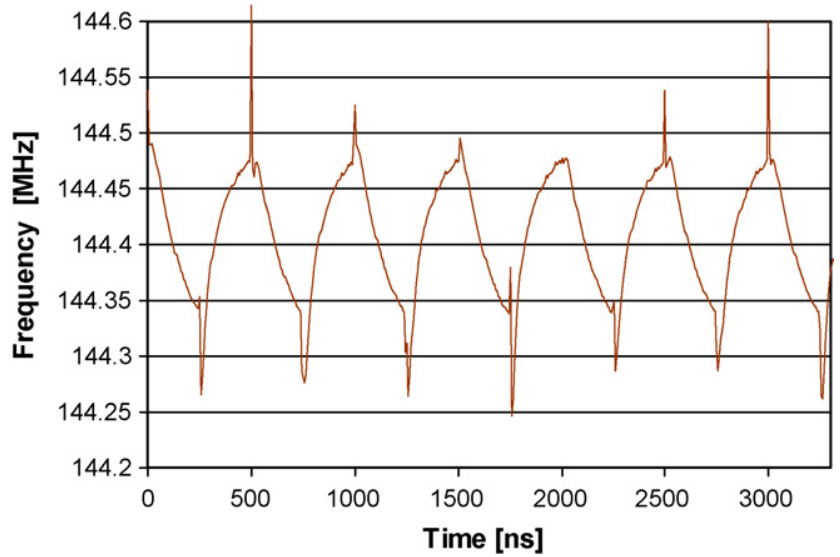

Fig. 5. Output frequency at 2 Mbps modulation rate at DATA simulation.

Carrier frequency vs parasitic load

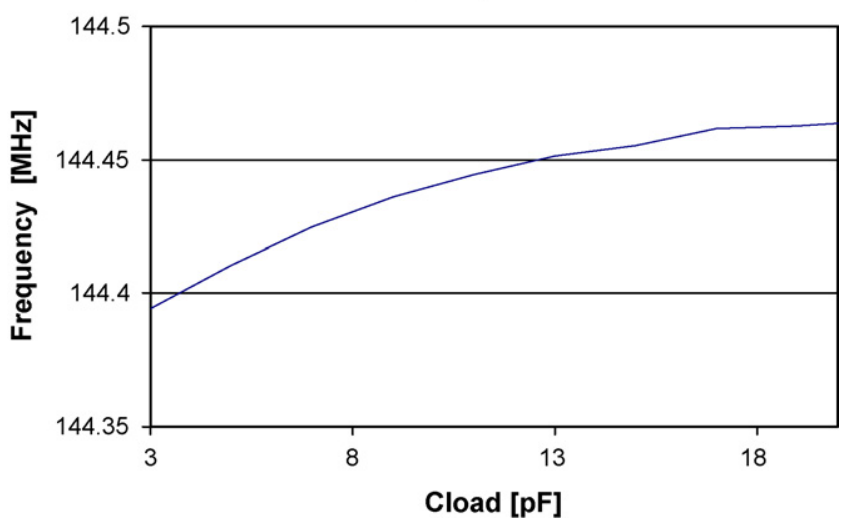

Fig. 6. Carrier sensitivity to parasitic load changes simulation.

ment algorithm. Note the exponential curves at each data edge. This is the consequence of the base capacitance being (dis)charged through the $R_{\text {data }}$ resistor. This RC time constant puts a limit on the maximum achievable data rate.

An important advantage of this transmitter topology is the insensitivity of the frequency vs. load capacitance variations (Fig. 6).

The change in frequency is $<0.15 \%$ for a change in capacitive load of $20 \mathrm{pF}$. This is a huge improvement compared to [13], where the output frequency is directly proportional to the output capacitive load.

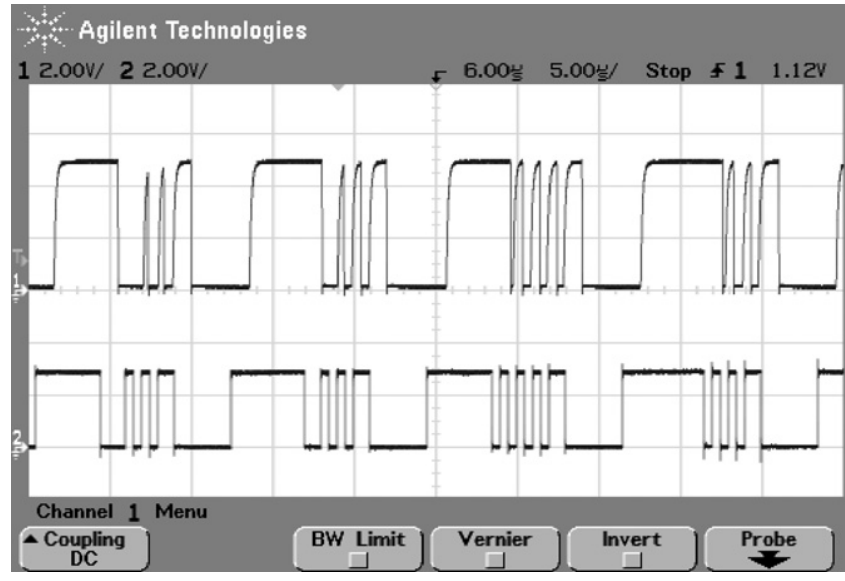

Fig. 7. Transmitted (bottom) and demodulated (top) data at 2 Mbps.

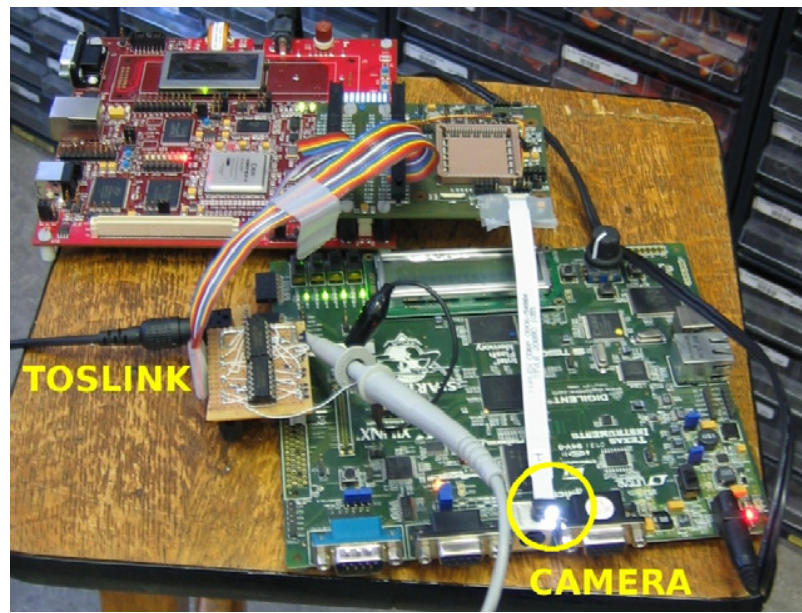

Fig. 8. VGA image sensor data serializer + optical link.

\section{Measurements}

The transmitter-receiver system functionality was initially characterized using the setup as described in [24]. This setup showed an unmodulated output power of $-18 \mathrm{dBm}$ at $50 \Omega$, for a supply voltage of $1.8 \mathrm{~V}$. The power consumption is $2 \mathrm{~mW}$ at a modulation rate of $2 \mathrm{Mbps}$, being equivalent to 15-20 VGA frames/s, using appropriate compression. This results in a FOM of $1 \mathrm{~nJ} / \mathrm{bit}$, which is lower than most state-of-the-art transmitter circuits, see Table 1. Fig. 7 depicts the data output of the receiver together with the data from the pseudorandom generator, showing that we are able to transmit and receive a data stream a 2 Mbps.

Table 1

Comparative table for capsule endoscopy image transmitter systems.

\begin{tabular}{|c|c|c|c|c|c|c|c|c|}
\hline & $\begin{array}{l}\text { Power cons. } \\
{[\mathrm{mW}]}\end{array}$ & $\begin{array}{l}\text { Supply voltage } \\
\text { [V] }\end{array}$ & $\begin{array}{l}\text { Carrier freq } \\
\text { [MHz] }\end{array}$ & $\begin{array}{l}\text { Datarate } \\
\text { [Mbps] }\end{array}$ & $\begin{array}{l}\text { Modulation } \\
\text { scheme }\end{array}$ & $\begin{array}{l}\text { Output power } \\
{[\mathrm{dBm}]}\end{array}$ & $\begin{array}{l}\text { Energy/bit } \\
\text { [nJ/bit] }\end{array}$ & $\begin{array}{l}\text { Transmitter } \\
\text { efficiency }\end{array}$ \\
\hline Zarlink Tx in M2A capsule & 5.2 & $2.6-3.2$ & $403 / 434$ & 2.7 & MSK & - & 1.9 & - \\
\hline Zarlink ZL70101 [15] & 17.5 & $2.5-3.2$ & $403 / 434$ & 0.8 & 2FSK/4FSK & -4.5 & 21.9 & $2.03 \%$ \\
\hline Nordic nRF2401 [16] & 37.8 & $1.9-3.6$ & $2400 / 2500$ & 1 & GFSK & 0 & 37.8 & $2.65 \%$ \\
\hline Shen et al. (2005) [17] & 4 & 1.85 & 416 & 2 & BFSK & -24 & 2.0 & $0.10 \%$ \\
\hline Boom et al. (2004) [18] & 1.8 & 0.9 & 433 & 0.1 & BFSK & -10 & 18.0 & $5.56 \%$ \\
\hline Chi et al. (2007) [19] & 7.9 & 2.5 & 2400 & 1 & ASK & -23 & 7.9 & $0.06 \%$ \\
\hline Itoh et al. (2006) [20] & 1.4 & 2 & 20 & 2.5 & BPSK & - & 0.6 & - \\
\hline Liu et al. (2007) [21] & 19.5 & 1.5 & 400 & 1.5 & FSK & -9 & 13.0 & $0.65 \%$ \\
\hline Hancock et al. (2007) [22] & 9.7 & 1.8 & $1350-1750$ & 2 & FSK & 3 & 4.9 & $20.57 \%$ \\
\hline Turgis et al. (2007) [14] & 6 & 1.7 & 120 & 1 & FSK & - & 6.0 & - \\
\hline This work & 2 & 1.8 & 144 & 2 & FSK & -18 & 1.0 & $0.79 \%$ \\
\hline
\end{tabular}

Please cite this article in press as: J. Thoné, et al., Design of a 2 Mbps FSK near-field transmitter for wireless capsule endoscopy, Sens. Actuators A: Phys. (2009), doi:10.1016/j.sna.2008.11.027 


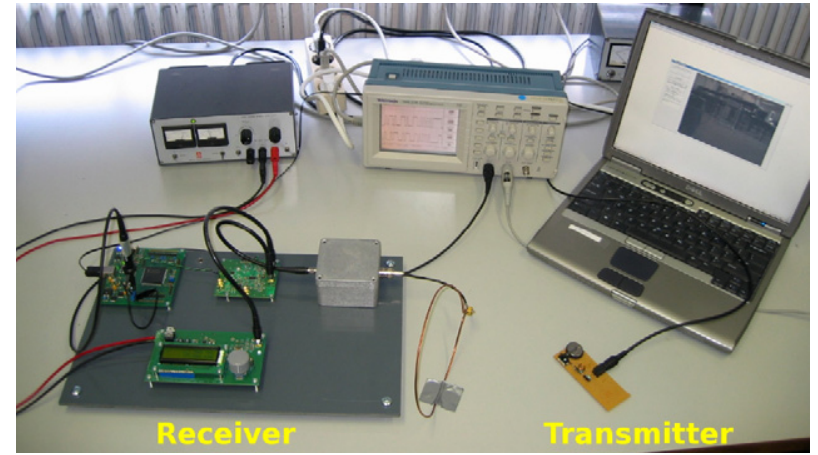

Fig. 9. Complete receiver setup with battery powered FSK transmitter, receiving serial image data from the optical link.

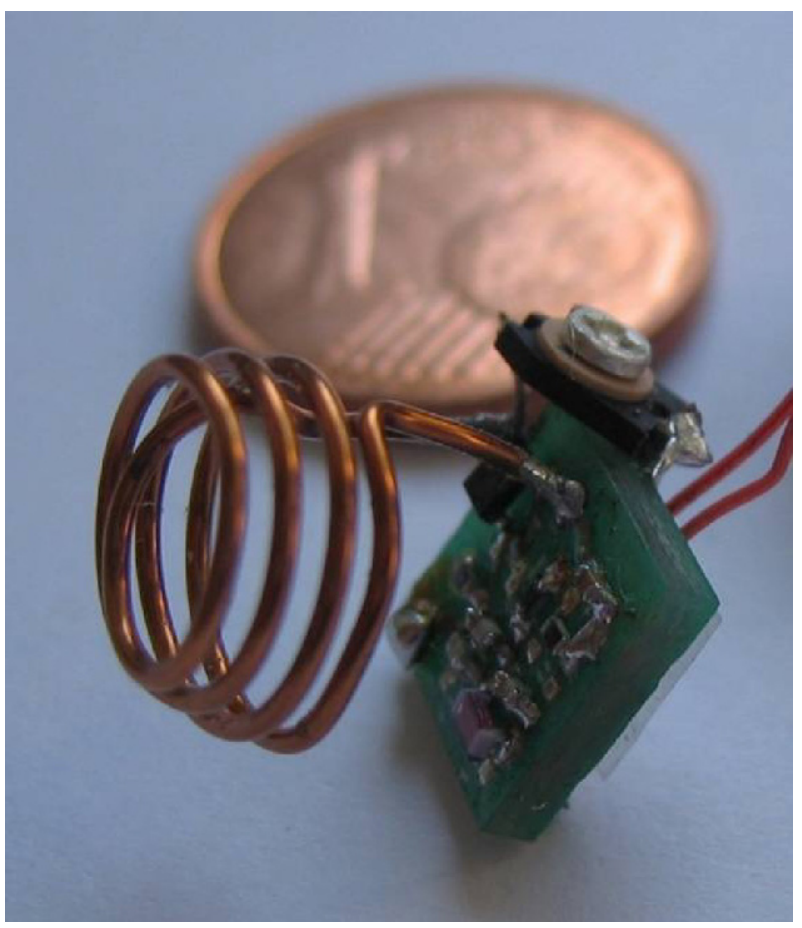

Fig. 10. Assembled FSK transmitter with coil antenna.

A more qualitative test setup was conceived in the meantime, depicted in Figs. 8 and 9. The transmitted data is generated from a Micron MT9V013 VGA image sensor (Fig. 8 top board+sensor were provided by Vector partner Neuricam). As 30 VGA frames per second are continuously provided, and maximally 2 Mbps can be handled by the transmitter, only one out of 30 frames is buffered in a DRAM, and then slowly released from the buffer (Fig. 8, bottom board). This leads to a data rate of $1.5 \mathrm{Mbps}$, or about $0.5 \mathrm{VGA}$ fps which is fed to the transmitter. To avoid ground loops, or influence/crosstalk between the transmitter and the data generation board, a galvanic isolation between both is mandatory. A TOSLINK optical transmitter and receiver (TOTX147 and TORX147) were used for this purpose, allowing complete galvanic isolation between the data source and the transmitter.

Fig. 9 depicts the functional transmitter/receiver setup, together with the GUI showing the received picture. Transmitter and receiver are at a distance of about $20 \mathrm{~cm}$. The big difference between [24] and this setup is the use of real VGA image data, as well as the use of near field instead of far-field antennas. For these tests, the transmitter was battery powered by a $3 \mathrm{~V}$ coin cell.
The transmitter measures only $0.42 \mathrm{~cm}^{2}$ which easily enables integration into an endoscopic capsule. Fig. 10 depicts the assembled FSK transmitter.

\section{Conclusion}

A simple, high data rate and low power transmitter is designed, fabricated and measured. It enables a 4-5 times higher image resolution (VGA instead of QVGA) and 15-17 fps for inductively powered endoscopic capsules. This development greatly improves the acceptation level by the surgeon, and will help to achieve correct diagnosis for GI tract diseases.

Future improvements and measurements consist of improving the Tx-Rx antenna system, full integration with a wireless powering module and quantitative tests of the transmitter module, like Bit Error Rate (BER) vs. distance and BER vs. transmission power.

More tests need be done on the functional integration of an inductive powering module and transmitter, especially with regards to harmonics of the inductive power link disturbing the transmitted FSK spectrum.

\section{Acknowledgments}

This work is supported by the European Community, within the 6th Framework Programme, through the Vector project (contract number 0339970). The authors wish to thank the project partners and the funding organization.

\section{References}

[1] C. Mc Caffrey, O. Chevalerias, C. O'Mathuna, K. Two, Swallowable-capsule technology, Pervasive Comput. (January) (2008) 23-29.

[2] M. Mylonaki, A. Fritscher-Ravens, P. Swain, Wireless capsule endoscopy: a comparison with push enteroscopy in patients with gastroscopy and colonoscopy negative gastrointestinal bleeding, Gut 52 (2003) 1122-1126.

[3] B. Lenaerts, R. Puers, An inductive power link for a wireless endoscope, Biosens. Bioelectron. 22 (7) (2007) 1390-1395.

[4] http://www.olympus-europa.com/.

[5] D. Turgis, R. Puers, Image compression in video radio transmission for capsule endoscopy, Sens. Actuators A: Phys. 123-124 (September) (2005) 129-136.

[6] C.C. Johnson, A.W. Guy, Nonionizing electromagnetic wave effects in biological materials and systems, Proc. IEEE 60 (1972) 692-720.

[7] L.C. Chirwa, P.A. Hammond, S. Roy, D.R.S. Cumming, Electromagnetic radiation from ingested sources in the human intestine between $150 \mathrm{MHz}$ and $1.2 \mathrm{GHz}$, IEEE Trans. Biomed. Eng. 50 (4) (2003) 484-492.

[8] L. Wang, T.D. Drysdale, D.R.S. Cumming, In-situ characterization of two wireless transmission schemes for ingestible capsules, IEEE Trans. Biomed. Eng. 54 (11) (2007) 2020-2027.

[9] US frequency allocation chart: http://www.fcc.gov/mb/engineering/usallochrt. pdf.

[10] EU frequency allocation overview: http://www.ero.dk/doc98/official/pdf/ rep025.pdf.

[11] J.M. Taylor, A. Terzuoli, On the concept of near field radar cross section, IEEE Antennas Propag. Soc. Int. Symp. 2 (July) (1997) 1172-1175.

[12] S. Chen, V. Thomas, Optimization of inductive RFID technology, IEEE Int. Symp. Electron. Environ. (May) (2001) 82-87.

[13] R.S. Mackay, B. Jackobson, Endoradiosonde, Nature 179 (June (4572)) (1957) 1239-1240.

[14] D. Turgis, R. Puers, A low power radio telemetry achieving very high data rates at biocompatible frequencies, Proc. Transducers Eurosensors (June)(2007) 1931-1934.

[15] Datasheet ZL70101: http://assets.zarlink.com/DS/zarlink_ZL70101_APR_07.pdf.

[16] Product page Nordic nRF2401A: http://www.nordicsemi.com/index.cfm?obj= product\&act=display \&pro=64.

[17] M. Shen, C. Lee, J. Bor, A $4.0 \mathrm{~mW}$ 2-Mbps programmable BFSK transmitter for capsule endoscope applications, Proc. IEEE ASSCC (November) (2005) 245-248.

[18] N. Boom, W. Rens, J. Crols, A $5.0 \mathrm{~mW}$ 0dBm FSK transmitter for $315 / 433 \mathrm{MHz}$ ISM applications in $0.25 \mu \mathrm{m}$ CMOS, Proc. 29th ESSCIRC (September) (2004) 199-202.

[19] B. Chi, J. Yao, S. Han, X. Xie, G. Li, Z. Wang, Low power transceiver analog front-end circuits for bidirectional high data rate wireless telemetry in medical endoscopy applications, IEEE Trans. Biomed. Eng. 54 (July (7)) (2007) 1291-1299.

[20] S. Itoh, S. Kawahito, S. Terakawa, A $2.6 \mathrm{~mW} 2$ fps QVGA CMOS one-chip wireless camera with digital image transmission function for capsule endoscopes, Proc. ISCAS 2006 (May) (2006) 3353-3356. 
[21] Y. Liu, T. Lin, An energy-efficient 1.5 Mbps wireless FSK transmitter with a deltasigma modulated phase rotator, Proc. 33rd ESSCIRC 2007 (September) (2007) 488-491.

[22] T. Hancock, M. Straayer, A. Messier, A Sub- $10 \mathrm{~mW} 2 \mathrm{Mbps}$ BFSK transceiver at 1.35 to $1.75 \mathrm{GHz}, 2007$ RFIC Symp. (June) (2007) 97-100.

[23] http://www.mentor.com/products/ic_nanometer_design/custom_design simulation/eldo/.

[24] J. Thone, D. Turgis, R. Carta, R. Puers, Design of an inductively powered 2 mbps FSK transmitter for wireless capsule endoscopy, Proc. Eurosensors XXII 2008 (September) (2008) 374-377.

[25] R. Carta, B. Lenaerts, J. Thone, R. Puers, G. Tortora, P. Valdastri, A. Menciassi, P. Dario, Wireless power supply as enabling technology towards active locomotion in capsular endoscopy, Proc. Eurosensors XXII 2008 (September) (2008) 1369-1372.

\section{Biographies}

Jef Thoné received the bachelor degree in automotive technology in 1999 from the Hogeschool voor Wetenschap en Kunst in Schaarbeek, Belgium. In 2002 he received his master of engineering degree in electronics at the Karel de Grote Hogeschool in Hoboken, Belgium.

From 2002 to 2006 he was employed as ASIC designer and project manager at Melexis Belgium, where he cooperated in the analog design of several automotive sensor interface ASICs.

In October 2006 he joined the Sensor Systems Group of MICAS. His PhD research focuses on high-data rate biocompatible telemetry systems. His research interests include sensor interfacing, analog IC and discrete design, FPGA and microcontroller interfacing, electronic system design and prototyping.

Soheil Radiom joined MICAS-ESAT at Katholieke Universiteit Leuven-Belgium from December 2006 for his PhD right after he got his MSc in electrical engineering from Ferdowsi University of Mashad, Iran. The subject of his MSc thesis was LowOversampling Continuous-Time Delta-Sigma Modulators while he was involved in Digital to Analog converter design in parallel. For his $\mathrm{PhD}$ he is working as a research assistant on integrated on-chip antennas, ultra wide-band antennas and ultra lowpower low-voltage analog circuits for RFID and wireless sensor network applications.

David Turgis was born in Saint-Lô, France in 1978. He received his master diploma of electronics from the Ecole Francaise d'Eléctronique et d'informatique-EFREI-in 2001. Currently, he is PhD student at the Katholieke Universiteit Leuven. He is dealing with data transmission for swallowable video capsules. His research interests are radio transmission through the human body, miniaturization and image data processing.

Riccardo Carta was born in Cagliari, Italy, in 1978.

In 2004 he received the degree of MSc in electrical engineering, with major in microelectronics, from the University of Cagliari, Italy.

Currently, he is a research assistant at the MICroelectronics And Sensors (MICAS) group of the Katholieke Universiteit Leuven, Belgium, where he is working towards a $\mathrm{PhD}$ degree. His main research interests are in the area of wireless power and data transmission for biomedical applications.
Georges G.E. Gielen received the MSc and PhD degrees in electrical engineering from the Katholieke Universiteit Leuven, Belgium, in 1986 and 1990, respectively. He currently is a full professor at the Katholieke Universiteit Leuven. His research interests are in the design of analog and mixed-signal integrated circuits, and especially in analog and mixed-signal CAD tools and design automation (modeling, simulation and symbolic analysis, analog synthesis, analog layout generation, analog and mixed-signal testing). He is coordinator or partner of several (industrial) research projects in this area, including several European projects (EU, MEDEA, ESA). He has authored or co-authored five books and more than 300 papers in edited books, international journals and conference proceedings. He regularly is a member of the Program Committees of international conferences (DAC, ICCAD, ISCAS, DATE CICC.), and served as General Chair of the DATE conference in 2006 and of the International Conference on Computer-Aided Design in 2007. He serves regularly as member of editorial boards of international journals (IEEE Transactions on Circuits and Systems, Springer international journal on Analog Integrated Circuits and Signal Processing, Elsevier Integration). He received the 1995 Best Paper Award in the John Wiley international journal on Circuit Theory and Applications, and was the 1997 Laureate of the Belgian Royal Academy on Sciences, Literature and Arts in the discipline of Engineering. He received the 2000 Alcatel Award from the Belgian National Fund of Scientific Research for his innovative research in telecommunications, and won the DATE 2004 Best Paper Award. He is a Fellow of the IEEE, served as elected member of the Board of Governors of the IEEE Circuits And Systems (CAS) society and as Chairman of the IEEE Benelux CAS chapter. He served as the President of the IEEE Circuits And Systems (CAS) Society in 2005. He was elected DATE Fellow in 2007, and received the IEEE Computer Society Outstanding Contribution Award and the IEEE Circuits and Systems Society Meritorious Service Award in 2007

Robert Puers received his BS degree in electrical engineering in Ghent in 1974, and his MS degree at the Katholieke Universiteit Leuven in 1977, where he obtained his $\mathrm{PhD}$ in 1986

In 1986 he became Director (NFWO) of the clean room facilities for silicon and hybrid circuit technology at the ESAT-MICAS laboratories of the same University. He was a pioneer in the European research efforts in silicon micromachined sensors, MEMS and packaging techniques, for biomedical implantable systems as well as for industrial devices. In addition, his general interest in low power telemetry systems, with emphasis on low power intelligent interface circuits and on inductive power and communication links has promoted the research of the ESAT-MICAS laboratory to international recognition.

At present, he is a full professor at the K.U. Leuven, teaching courses in 'Microsystems and Sensors', in 'Biomedical Instrumentation and Stimulation', in 'Biomedica Equipment and Regulations', in 'Production techniques for microelectronics', and basic courses in 'Electronics, System Control and Information Technology'. He is the author or co-author of more than 400 papers on biotelemetry, sensors, MEMS and packaging in reviewed journals or international conferences.

He is a Fellow of the Institute of Physics (UK), council member of the Internationa Microelectronics and Packaging Society (IMAPS), member of the Electron Device Society (EDS) and many others.

He is Editor-in-Chief of the IOP Journal of Micromechanics and Microengineering, and acted as the General Program Chairman for Transducers'07 in Lyon. 